\title{
Nuclear enhancement and suppression of diffractive structure functions at high energies
}

\author{
H. Kowalski, ${ }^{1}$ T. Lappi ${ }^{2}$ C. Marquet, ${ }^{2,3}$ and R. Venugopalan ${ }^{4}$ \\ ${ }^{1}$ Deutsches Elektronen-Synchrotron DESY, 22607 Hamburg, Germany \\ ${ }^{2}$ Institut de Physique Théorique, Bât. 774, CEA/DSM/Saclay, 91191 Gif-sur-Yvette, France \\ ${ }^{3}$ Department of Physics, Columbia University, New York, NY 10027, USA \\ ${ }^{4}$ Physics Department, Brookhaven National Laboratory, Upton, NY 11973, USA
}

\begin{abstract}
We compute diffractive structure functions for both protons and nuclei in the framework of Color Glass Condensate models with impact parameter dependence. These models have previously been shown to provide good agreement with inclusive $F_{2}$ measurements and exclusive vector meson measurements at HERA. For nuclei, they provide good (parameter free) agreement with the inclusive $F_{2}$ data. We demonstrate good agreement of our computations with HERA measurements on inclusive diffraction. We extend our analysis to nuclei and predict the pattern of enhancement and suppression of the diffractive structures functions that can be measured at an Electron Ion Collider. We discuss how the impact parameter dependence crucially affects our analysis, in particular for large invariant masses at fixed $Q^{2}$.

PACS numbers: 13.60.Hb,24.85.+p
\end{abstract}

\section{INTRODUCTION}

The discovery that about 15\% of Deeply Inelastic Scattering (DIS) events at HERA are diffractive events [1, 2] has focused attention on the nature of hard diffractive scattering in QCD at collider energies. In particular, it is observed that the ratio of diffractive to inclusive cross-sections is nearly independent of the energy [3, 4], and that both cross-sections display geometric scaling [5, 6, 7]. It was noted some time ago that these features could be understood [8] in a simple model, the Golec-Biernat-Wusthoff (GBW) dipole model [9], which incorporated the physics of QCD saturation at high energies. The saturation of parton densities is due to non-linear multi-parton effects such as recombination and screening which deplete the gluon density at small $x$ [10]. These non-linear effects are large for modes in the hadronic wavefunctions with transverse momenta $k_{\perp} \lesssim Q_{\mathrm{s}}$, where $Q_{\mathrm{s}}(x)$, appropriately called the saturation scale, is a scale generated by the multiparton dynamics. Though the Golec-Biernat-Wusthoff model explains qualitative features of the inclusive and diffractive data, it fails in detailed comparisons to the data. This is primarily because, except for the quark masses, the model does not contain geometric scaling violations, such as for instance the bremsstrahlung limit of perturbative QCD (pQCD) that applies to small dipoles of transverse size $r \ll 1 / Q_{\mathrm{s}}(x)$.

The GBW model is significantly improved by including the appropriate DGLAP behavior for dipoles with small transverse sizes 11]. This "DGLAP improved" dipole model arises naturally in the classical effective theory of the Color Glass Condensate (CGC) [12, 13, 14]. For instance, in the CGC [15, 16], one obtains the well known expression [17, 18] for the inclusive virtual photon hadron cross section

$$
\sigma_{L, T}^{\gamma^{*} p}=\int \mathrm{d}^{2} \mathbf{r}_{T} \int_{0}^{1} \mathrm{~d} z\left|\Psi_{L, T}^{\gamma^{*}}\right|^{2} \int \mathrm{d}^{2} \mathbf{b}_{T} \frac{\mathrm{d} \sigma_{\mathrm{dip}}^{\mathrm{p}}}{\mathrm{d}^{2} \mathbf{b}_{T}},
$$

where $\left|\Psi_{L, T}^{\gamma^{*}}\left(\mathbf{r}_{T}, z, Q\right)\right|^{2}$ represents the probability for a virtual photon to produce a quark-anti-quark pair of size $r=\left|\mathbf{r}_{T}\right|$ and $\frac{\mathrm{d} \sigma_{\mathrm{dip}}^{\mathrm{p}}}{\mathrm{d}^{2} \mathbf{b}_{T}}\left(\mathbf{r}_{T}, x, \mathbf{b}_{T}\right)$ denotes the dipole cross section for this pair to scatter off the target at an impact parameter $\mathbf{b}_{T}$. The former is well known from QED, while the latter represents the dynamics of QCD scattering at small $x$. In the "classical" limit without high energy evolution effects, the dipole cross section can be written as

$$
\frac{\mathrm{d} \sigma_{\mathrm{dip}}^{\mathrm{p}}}{\mathrm{d}^{2} \mathbf{b}_{T}}=2\left[1-\exp \left(-r^{2} F(x, r) T_{p}\left(\mathbf{b}_{T}\right)\right)\right],
$$

where $T_{p}\left(\mathbf{b}_{T}\right) \sim \exp \left(-\frac{b^{2}}{2 B_{\mathrm{G}}}\right)$ is the impact parameter profile function in the proton, normalized as $\int d^{2} \mathbf{b}_{T} T_{p}\left(\mathbf{b}_{T}\right)=1$ and $F$ is proportional to the DGLAP evolved gluon distribution [1]

$$
F\left(x, r^{2}\right)=\frac{\pi^{2}}{2 N_{\mathrm{c}}} \alpha_{\mathrm{s}}\left(\mu_{0}^{2}+\frac{C}{r^{2}}\right) x g\left(x, \mu_{0}^{2}+\frac{C}{r^{2}}\right) .
$$

In general, the dipole cross section can range from 0 in the $r \rightarrow 0$ color transparency limit to 2 , the maximal unitarity bound. The saturation scale $Q_{\mathrm{s}}$ characterizes the qualitative change between these regimes; following [19] we shall define here $Q_{\mathrm{s}}$ as the solution of

$$
\frac{\mathrm{d} \sigma_{\operatorname{dip}}}{\mathrm{d}^{2} \mathbf{b}_{T}}\left(x, r^{2}=1 / Q_{\mathrm{s}}{ }^{2}\left(x, \mathbf{b}_{T}\right)\right)=2\left(1-e^{-1 / 4}\right)
$$

Note that our definition is completely model independent and can be applied to any sensible parametrization of the dipole cross section. The definition (4) applied to a Gaussian dipole cross section gives the saturation scale of the 
GBW model, but it differs slightly from the convention in Ref. 20], where the saturation criterion was taken as $\frac{\mathrm{d} \sigma_{\mathrm{dip}}}{\mathrm{d}^{2} \mathbf{b}_{T}}=2\left(1-e^{-1 / 2}\right)$ at $r^{2}=2 / Q_{\mathrm{s}}{ }^{2}$. The dipole cross section in Eq. (2) was implemented in the impact parameter saturation model (IPsat) [20] where the parameters $\mu_{0}$, $C$, and $B_{\mathrm{G}}$ (as well as two other parameters characterizing the initial condition for the DGLAP evolution) are fit to reproduce the HERA data on the inclusive structure function $F_{2}$.

The form of the IPsat dipole cross section in Eq. (2) is applicable when leading logarithms in $Q^{2}$ dominate over leading logarithms in $x$. At very small $x$, quantum evolution in the CGC describes both the dilute bremsstrahlung limit of linear small $x$ evolution as well as nonlinear RG evolution at high parton densities [15]. The essential dynamics of this small $x$ evolution are combined with a more realistic $b$-dependence in the bCGC model [21, 22]. The model is formulated in terms of an explicit $x$-dependent saturation scale that we shall denote by $Q_{\mathrm{s}}^{\prime}$ to distinguish it from our model independent definition (4). The dipole cross section has the form

$$
\begin{aligned}
\frac{\mathrm{d} \sigma_{\mathrm{dip}}^{\mathrm{p}}}{\mathrm{d}^{2} \mathbf{b}_{T}} & =2 \mathcal{N}_{0}\left(\frac{r Q_{\mathrm{s}}^{\prime}}{2}\right)^{2\left(\gamma_{s}+\frac{1}{\kappa \lambda Y} \ln \left(\frac{2}{r Q_{\mathrm{s}}^{\prime}}\right)\right)} & & \text { for } r Q_{\mathrm{s}}^{\prime} \leq 2 \\
& =2-2 \exp \left(-A \ln ^{2}\left(B r Q_{\mathrm{s}}^{\prime}\right)\right) & & \text { for } r Q_{\mathrm{s}}^{\prime}>2 .
\end{aligned}
$$

The coefficients $A$ and $B$ in the second line of this equation can be determined uniquely from the condition that $\frac{\mathrm{d} \sigma_{\text {dip }}^{\mathrm{p}}}{\mathrm{d}^{2} \mathbf{b}_{T}}$ and its first derivative with respect to $r Q_{\mathrm{s}}^{\prime}$ are continuous across $r Q_{\mathrm{s}}^{\prime}=2$. Here $Y=\ln (1 / x)$ is the rapidity, while $\gamma_{s}=0.628$ and $\kappa=9.9$ (which quantifies the geometric scaling violations in (5)) are obtained from the leading logarithmic BFKL dynamics [23]. The impact parameter dependence of the proton saturation scale is introduced into the bCGC model in the form

$$
Q_{\mathrm{s}}^{\prime}(x, b)=\left(\frac{x_{0}}{x}\right)^{\frac{\lambda}{2}}\left[\exp \left(-b^{2} / 2 B_{\mathrm{CGC}}\right)\right]^{\frac{1}{2 \gamma_{s}}} \mathrm{GeV} .
$$

After choosing $\mathcal{N}_{0}=0.7$, the parameters $\lambda, x_{0}$ and $B_{\text {CGC }}$ are fit to the data. We will discuss the impact parameter dependence of the dipole cross-section and the saturation scale further in the next section. We must emphasize that the saturation scale $Q_{\mathrm{s}}$ is conceptually the same as $Q_{\mathrm{s}}^{\prime}$ and their numerical values are of the same order, but we differentiate between them in order to maintain our model independent definition (4) and the original parametrization of the bCGC model.

Both the IPsat model and the bCGC model provide excellent fits to a wide range of HERA data for $x \leq$ 0.01 22, 24]. In Ref. 19], we discussed the possibility that DIS off nuclei can distinguish respectively between these "classical CGC" and "quantum CGC" motivated models. Our discussion in that paper addressed the $A$ dependence of the nuclear saturation scale and fits to the available nuclear DIS inclusive data. We also addressed elastic scattering of $q \bar{q}$ dipoles of nuclei but our analysis was incomplete because a more complete picture of

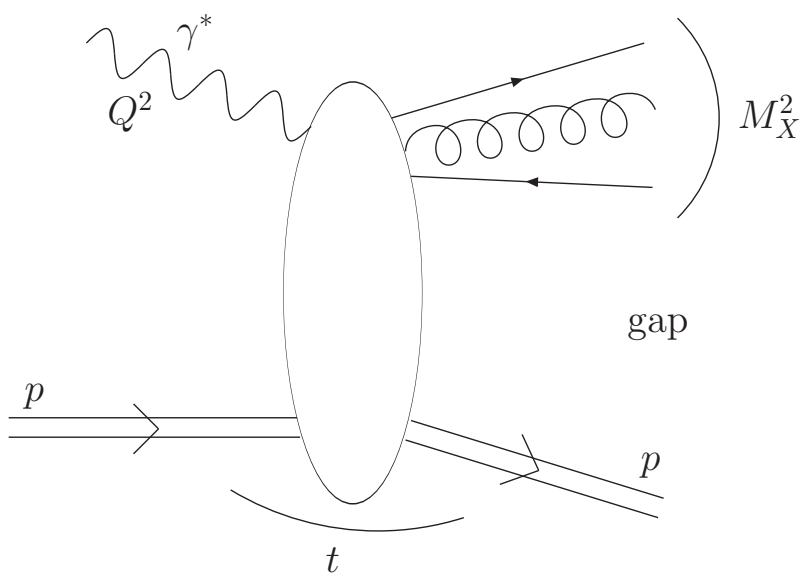

FIG. 1: Kinematics of diffractive DIS.

diffraction requires that one consider the diffractive scattering off nuclei of higher Fock states as well - at least of the $q \bar{q} g$ Fock state. This shortcoming is addressed in the present paper. We note that there have been several discussions of diffractive scattering on nuclei in the literature [25, 26, 27, 28, 29, 30, 31, 32, 33, 34, 35]. We will later compare and contrast our results with those studies that overlap with ours.

The paper is organized as follows. In the next section, we will introduce the kinematics for diffractive deep inelastic scattering and the relevant formulae to compute diffractive structure functions in the dipole model. We will discuss how the $q \bar{q}$ and $q \bar{q} g$ dipoles, the dominant contributions at present collider energies, contribute to the diffractive cross-section. Particular attention will be paid to the impact parameter dependence of the crosssections. In Sec. III, we will discuss a comparison of the different CGC based models to the HERA diffractive data. This analysis is extended to nuclei in Sec. IV. The "breakup" and "non breakup" events discussed in Ref. [19] for $q \bar{q}$ dipoles are applied to the $q \bar{q} g$ dipoles as well. We study the nuclear enhancement and suppression of diffractive cross-sections in the different models and assess their predictive power. In the final section we compare our results with those existing in the literature and outline future research on this topic.

\section{COMPUTING THE DIFFRACTIVE STRUCTURE FUNCTION}

In the dipole picture of diffractive DIS, the virtual photon fluctuates into a colorless parton Fock state, which interacts elastically with the proton or nucleus. In the final state, the latter remains intact, while (for inclusive diffraction), the parton Fock state fragments into hadrons with an invariant mass $M_{X}$. In addition to the usual DIS invariants $x$ and $Q^{2}$, inclusive diffractive scattering can be fully characterized by two other invariants $\beta$ and $t$. Here $t=-\left(P-P^{\prime}\right)^{2}$, where $P^{\mu}$ and $P^{\prime \mu}$ denote 
the four-momenta of the incoming and outgoing hadron, and $\beta=Q^{2} /\left(Q^{2}+M_{X}^{2}\right)$, with $0<\beta<1$. Note that if $Y=\ln (1 / x)$ is the relative rapidity between the electron and the hadron, $\ln (1 / \beta)$ is the rapidity interval between the electron and the hadronic fragments $X$ of the Fock state. The rapidity gap between $X$ and the proton or nucleus is expressed as $Y_{\mathbb{P}}=\ln \left(1 / x_{\mathbb{P}}\right)$; therefore, by definition, $Y=\ln (1 / \beta)+Y_{\mathbb{P}}$, or $x_{\mathbb{P}}=x / \beta$. The kinematics is illustrated in Fig. 1.

The parton Fock state could be a $q \bar{q}$ dipole or higher Fock states which involve emission of one or more gluons. Naively, these higher Fock states are suppressed by higher powers of $\alpha_{\mathrm{s}}$; however, in the limits of large $Q^{2}$ or small $\beta$, gluon emissions are accompanied by large logarithms in $Q^{2}$ and $\beta$ respectively that compensate the powers of the coupling. These factors then have to be resummed to obtain the diffractive cross-sections in the appropriate kinematic domains. The powers of $\alpha_{\mathrm{s}} \ln 1 / \beta$ can in principle be resummed in the dipole picture by using the Kovchegov-Levin equation 36 in the mean-field approximation, and the approach of [37] beyond. In practice one must require $x_{\mathbb{P}}<0.01$ for the rapidity gap to be large enough to be clearly detectable and to be in the domain of validity of the dipole model. Thus in the kinematical regime of HERA (and realistic future DIS experiments) $\ln 1 / \beta$ is not very large and we shall only take into account the contribution of the $q \bar{q}$ and $q \bar{q} g$ components in the present work.

In diffractive scattering, contrary to inclusive scattering, one is really computing the square of an amplitude - with interference terms. As a consequence, the square of the photon wave function cannot be factorized from the cross section. Introducing the auxiliary notation

$$
\Phi_{n}=\int \mathrm{d}^{2} \mathbf{b}_{T}\left[\int_{0}^{\infty} \mathrm{d} r r K_{n}(\varepsilon r) J_{n}(k r) \frac{\mathrm{d} \sigma_{\mathrm{dip}}}{\mathrm{d}^{2} \mathbf{b}_{T}}\left(\mathbf{b}_{T}, r, x_{\mathbb{P}}\right)\right]^{2}
$$

the $q \bar{q}$ components of the diffractive structure function. can be expressed as

$$
\begin{aligned}
x_{\mathbb{P}} F_{T, q \bar{q}}^{\mathrm{D}}\left(x_{\mathbb{P}}, \beta, Q^{2}\right) & =\frac{N_{\mathrm{c}} Q^{4}}{16 \pi^{3} \beta} \sum_{f} e_{f}^{2} \int_{z_{0}}^{1 / 2} \mathrm{~d} z z(1-z) \\
\times & {\left[\varepsilon^{2}\left(z^{2}+(1-z)^{2}\right) \Phi_{1}+m_{f}^{2} \Phi_{0}\right] } \\
x_{\mathbb{P}} F_{L, q \bar{q}}^{\mathrm{D}}\left(x_{\mathbb{P}}, \beta, Q^{2}\right) & =\frac{N_{\mathrm{c}} Q^{6}}{4 \pi^{3} \beta} \sum_{f} e_{f}^{2} \int_{z_{0}}^{1 / 2} \mathrm{~d} z z^{3}(1-z)^{3} \Phi_{0}
\end{aligned}
$$

with $\varepsilon^{2}=z(1-z) Q^{2}+m_{f}^{2}, k^{2}=z(1-z) M_{X}^{2}-m_{f}^{2}$ and $z_{0}=\left(1-\sqrt{1-4 m_{f}^{2} / M_{X}^{2}}\right) / 2$. In principle the measurable $|t|$ is bounded by a very small kinematical lower bound $|t|>\left(x_{\mathbb{P}} m_{N}\right)^{2}$ and both upper and lower experimental limits. Here we have, however, performed the $t$ integration from $-\infty$ to 0 , because this enables us write the above expressions in impact parameter space. Since the decrease of the structure functions with $t$ is (close to an) exponential, this approximation is reasonable. Note that we allow for an arbitrary impact parameter dependence, and thus there is no "diffractive slope" as a separate parameter.

For the $q \bar{q} g$ component of the structure function, there are essentially two approaches that have been used. The one derived in the large $Q^{2}$-limit in Ref. [38] gives

$$
\begin{aligned}
& x_{\mathbb{P}} F_{T, q \bar{q} g}^{\mathrm{D}(\mathrm{GBW})}\left(x_{\mathbb{P}}, \beta, Q^{2}\right)= \\
& \frac{\alpha_{\mathrm{s}} \beta}{8 \pi^{4}} \sum_{f} e_{f}^{2} \int \mathrm{d}^{2} \mathbf{b}_{T} \int_{0}^{Q^{2}} \mathrm{~d} k^{2} \int_{\beta}^{1} \mathrm{~d} z\{ \\
& k^{4} \ln \frac{Q^{2}}{k^{2}}\left[\left(1-\frac{\beta}{z}\right)^{2}+\left(\frac{\beta}{z}\right)^{2}\right] \\
& \times\left[\int_{0}^{\infty} \mathrm{d} r r \frac{\mathrm{d} \tilde{\sigma}_{\mathrm{dip}}}{\mathrm{d}^{2} \mathbf{b}_{T}}\left(\mathbf{b}_{T}, \mathbf{r}_{T}, x_{\mathbb{P}}\right)\right. \\
& \left.\left.K_{2}(\sqrt{z} k r) J_{2}(\sqrt{1-z} k r)\right]^{2}\right\} .
\end{aligned}
$$

In this limit, the $q \bar{q} g$-system is an adjoint representation $g g$ dipole, therefore, as in Ref. [39], we use here the dipole cross section in the adjoint representation, denoted by

$$
\frac{\mathrm{d} \tilde{\sigma}_{\mathrm{dip}}}{\mathrm{d}^{2} \mathbf{b}_{T}}=2\left[1-\left(1-\frac{1}{2} \frac{\mathrm{d} \sigma_{\mathrm{dip}}}{\mathrm{d}^{2} \mathbf{b}_{T}}\right)^{2}\right] .
$$

This large $N_{\mathrm{c}}$ expression, where the $g g$-system is treated as two fundamental representation $q \bar{q}$ dipoles, is consistent with the Balitsky-Kovchegov [40, 41, 42] mean field treatment of small $x$ evolution. The same is true of the $\beta \rightarrow 0$-limit (Eq. (12)) below, where the $q \bar{q} g$ system is also treated as two fundamental representation $q \bar{q}$ dipoles, but this time with different sizes. We will refer to Eq. (10) as the "GBW" $q \bar{q} g$-component (referring to [8]). Note that the form (11) differs from the one used in Ref. [8], where the adjoint dipole does not have the right saturation limit.

The other well studied case is the $\beta \rightarrow 0$ limit 33,43 , 44, 45, 46, 47]. In this limit (the notation "MS" below refers to the authors of [43]) the structure function again factorizes into the product of a photon wave function and the cross section for the $q \bar{q} g$-system to interact elastically (diffractively) with the target:

$$
\begin{gathered}
x_{\mathbb{P}} F_{T, q \bar{q} g}^{\mathrm{D}(\mathrm{MS})}\left(x_{\mathbb{P}}, \beta=0, Q^{2}\right)=\frac{C_{\mathrm{F}} \alpha_{\mathrm{s}} Q^{2}}{4 \pi^{4} \alpha_{\mathrm{em}}} \int \mathrm{d}^{2} \mathbf{r}_{T} \int_{0}^{1} \mathrm{~d} z \\
\left|\Psi_{T}^{\gamma^{*}}(r, Q, z)\right|^{2} \int \mathrm{d}^{2} \mathbf{b}_{T} A\left(r, x_{\mathbb{P}}, \mathbf{b}_{T}\right),
\end{gathered}
$$

with

$$
\begin{array}{r}
A\left(r, x_{\mathbb{P}}, \mathbf{b}_{T}\right)=\int \mathrm{d}^{2} \mathbf{r}_{T}^{\prime} \frac{\mathbf{r}_{T}{ }^{2}}{\mathbf{r}_{T}^{\prime 2}\left(\mathbf{r}_{T}-\mathbf{r}_{T}^{\prime}\right)^{2}}\left[\mathcal{N}\left(\mathbf{r}_{T}^{\prime}\right)\right. \\
\left.+\mathcal{N}\left(\mathbf{r}_{T}-\mathbf{r}_{T}^{\prime}\right)-\mathcal{N}\left(\mathbf{r}_{T}\right)-\mathcal{N}\left(\mathbf{r}_{T}^{\prime}\right) \mathcal{N}\left(\mathbf{r}_{T}-\mathbf{r}_{T}^{\prime}\right)\right]^{2}
\end{array}
$$




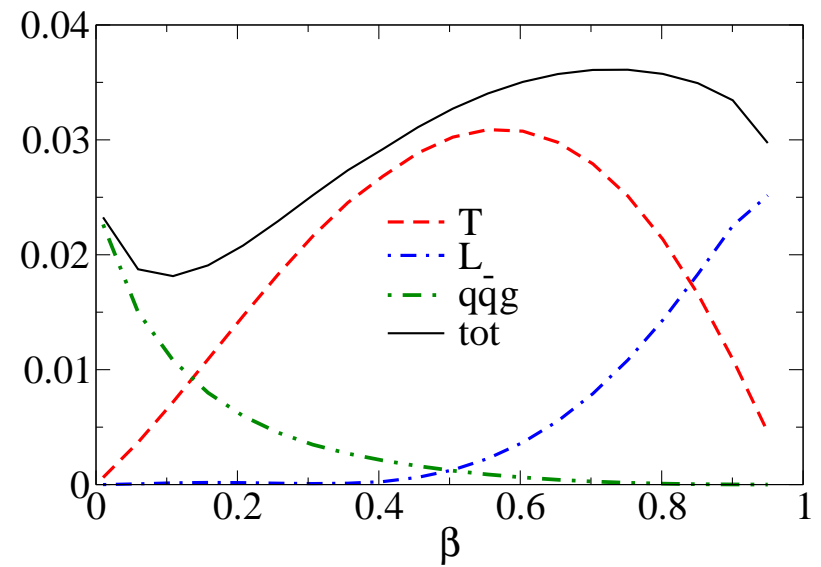

FIG. 2: $\beta$-dependence of the different contributions to the proton diffractive structure function at $Q^{2}=5 \mathrm{GeV}^{2}$ and $x_{\mathbb{P}}=10^{-3}$.

and $\mathcal{N}\left(r, x_{\mathbb{P}}, \mathbf{b}_{T}\right)=\frac{\mathrm{d} \sigma_{\mathrm{dip}}}{\mathrm{d}^{2} \mathbf{b}_{T}}\left(r, x_{\mathbb{P}}, \mathbf{b}_{T}\right) / 2$. The coupling constant $\alpha_{\mathrm{s}}$ in Eqs. (10) and (12) is treated here as a constant free parameter (independent of the DGLAP evolution momentum scale in the IPsat model). A more thorough study of the running coupling effects in this problem is an interesting question that is out of the scope of the present work.

Depending on the mass of the diffractive system $M_{X}$ or, equivalently, $\beta$, the diffractive structure function is dominated by either the $q \bar{q}$ or $q \bar{q} g$ Fock states. (See Ref. [48] for a general argument of the $\beta$ dependence.) Specifically, in the limit $\beta \rightarrow 1$, the dominant component, $F_{L, q \bar{q}}^{\mathrm{D}}$ is a longitudinally polarized $q \bar{q}$ system. At intermediate $\beta \sim 0.5$ the dominant component is a transversally polarized $q \bar{q}$ denoted here by $F_{T, q \bar{q}}^{\mathrm{D}}$. In the limit $\beta \rightarrow 0$ the invariant mass of the diffractive system is large, and this large phase space is filled by radiation of additional gluons, each of them being suppressed by $\alpha_{\mathrm{s}}$. This structure is illustrated in Fig. 2,

In Ref. [39], it was shown that the $\beta=0$ limit of Eq. (10), at $Q^{2} \rightarrow \infty$, approaches the result from Eq. (12). This therefore suggests the following interpolation formula between the two limits [39]:

$$
\begin{aligned}
& x_{\mathbb{P}} F_{T, q \bar{q} g}\left(x_{\mathbb{P}}, \beta, Q^{2}\right)= \\
& \frac{x_{\mathbb{P}} F_{T, q \bar{q} g}^{\mathrm{D}(\mathrm{GBW})}\left(x_{\mathbb{P}}, \beta, Q^{2}\right) \times x_{\mathbb{P}} F_{T, q \bar{q} g}^{\mathrm{D}(\mathrm{MS})}\left(x_{\mathbb{P}}, Q^{2}\right)}{x_{\mathbb{P}} F_{T, q \bar{q} g}^{\mathrm{D}(\mathrm{GBW})}\left(x_{\mathbb{P}}, \beta=0, Q^{2}\right)} .
\end{aligned}
$$

We also note the work in Refs. [30, 32, 49], which uses yet another prescription whose relation to ours is not very transparent.

\section{IMPACT PARAMETER DEPENDENCE}

We shall now discuss the $b$-dependence of the dipole cross-sections (see also e.g. Refs. [50, 51, 52]). Several
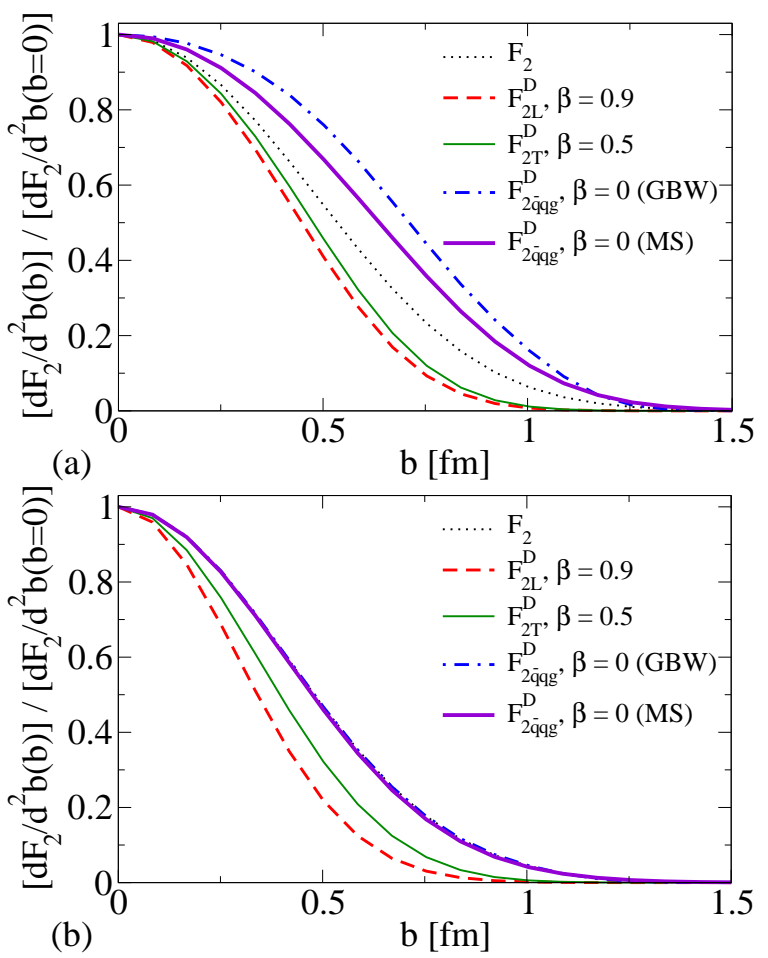

FIG. 3: b-dependence of the the inclusive structure function and different contributions to the diffractive structure function at $Q^{2}=1 \mathrm{GeV}^{2}$ (a) and $Q^{2}=100 \mathrm{GeV}^{2}$ (b) for $x=10^{-3}$ (inclusive) and $x_{\mathbb{P}}=10^{-3}$ (diffractive). In plot (b) $\left(Q^{2}=100 \mathrm{GeV}^{2}\right)$ the $b$-dependence of the inclusive cross section and $q \bar{q} g$-components are indistinguishable.

works on the subject (for example, Refs. [8, 9, 21, 35]) assume, explicitly or implicitly, a factorizable $\mathbf{b}_{T}$ dependence

$$
\frac{\mathrm{d} \sigma_{\mathrm{dip}}}{\mathrm{d}^{2} \mathbf{b}_{T}}\left(\mathbf{b}_{T}, \mathbf{r}_{T}, x\right)=2 \mathcal{N}\left(\mathbf{b}_{T}, \mathbf{r}_{T}, x\right)=2 T_{p}\left(\mathbf{b}_{T}\right) N\left(\mathbf{r}_{T}, x\right) .
$$

When considering diffractive scattering on protons, this is consistent with the exponential $t$ dependence observed in experiments, and in fact implies that $T_{p}\left(\mathbf{b}_{T}\right)$ is Gaussian.

In the IPsat model, in contrast to the factorization of the $b$-dependence in Eq. (15), the dependence of the dipole cross-section on impact parameter is as in Eq. (2), which equivalent to an impact parameter dependence of the saturation scale $Q_{\mathrm{s}}{ }^{2} \propto T_{p}\left(\mathbf{b}_{T}\right)$. In the IPsat model the impact parameter profile of the proton saturation scale is chosen to have the form

$$
T_{p}\left(\mathbf{b}_{T}\right)=\frac{1}{2 \pi B_{\mathrm{G}}} e^{-\frac{b^{2}}{2 B_{\mathrm{G}}}},
$$

which is normalized to unity. In the large $Q^{2}$-limit the cross section is dominated by small dipoles and one can expand the exponential of Eq. (2), and the dipole cross section becomes proportional to $T_{p}\left(\mathbf{b}_{T}\right)$. This corresponds to $\left\langle b^{2}\right\rangle=2 B_{\mathrm{G}}$, which can be interpreted as the average square gluonic radius in the proton. 
We shall now justify in the framework of the BK equation why we prefer [19, 20, 22] to incorporate the impact parameter dependence in the saturation scale $Q_{\mathrm{s}}$ instead of as a multiplicative factor. (As we shall see, this difference is more significant for diffractive as opposed to inclusive observables.) The fully impact parameter dependent BK equation for the dipole amplitude is

$$
\begin{gathered}
\partial_{y} \mathcal{N}_{\mathbf{x}_{T}, \mathbf{y}_{T}}=\frac{\alpha_{\mathbf{s}} N_{c}}{2 \pi^{2}} \int \mathrm{d}^{2} \mathbf{z}_{T} \frac{\left(\mathbf{x}_{T}-\mathbf{y}_{T}\right)^{2}}{\left(\mathbf{x}_{T}-\mathbf{z}_{T}\right)^{2}\left(\mathbf{y}_{T}-\mathbf{z}_{T}\right)^{2}} \\
{\left[\mathcal{N}_{\mathbf{x}_{T}, \mathbf{z}_{T}}+\mathcal{N}_{\mathbf{z}_{T}, \mathbf{y}_{T}}-\mathcal{N}_{\mathbf{x}_{T}, \mathbf{y}_{T}}-\mathcal{N}_{\mathbf{x}_{T}, \mathbf{z}_{T}} \mathcal{N}_{\mathbf{z}_{T}, \mathbf{y}_{T}}\right]}
\end{gathered}
$$

where $\mathbf{b}_{T}=\left(\mathbf{x}_{T}+\mathbf{y}_{T}\right) / 2$ and $\mathbf{r}_{T}=\mathbf{x}_{T}-\mathbf{y}_{T}$. This equation was studied numerically in Ref. [53] with the conclusions, a) that the $\mathbf{b}_{T}$ and $\mathbf{r}_{T}$ dependence of the amplitude does not factorize, and b) one obtains unphysically large power law tails in $\mathbf{b}_{T}$. Physically this is due to the fact that the BK equation does not include confinement effects that would cut off these tails. An approximation that removes the growth of the power law tail with increasing $y=\log (1 / x)$ is based on the argument that the dipole sizes $r^{\prime} \sim 1 / Q_{s}$ (which dominate the integration in (17)) are parametrically smaller than the typical scale $R_{p}$ for the variation of the amplitude with impact parameter $b$. One can thus approximate (note that the same approximation was already implied when writing Eq. (13))

$$
\begin{array}{r}
\partial_{y} \mathcal{N}_{\mathbf{b}_{T}, \mathbf{r}_{T}} \approx \frac{\alpha_{\mathrm{s}} N_{c}}{2 \pi^{2}} \int \mathrm{d}^{2} \mathbf{r}_{T}^{\prime} \frac{\mathbf{r}_{T}^{2}}{\mathbf{r}_{T}^{\prime 2}\left(\mathbf{r}_{T}-\mathbf{r}_{T}^{\prime}\right)^{2}} \\
{\left[\mathcal{N}\left(\mathbf{b}_{T}, \mathbf{r}_{T}^{\prime}\right)+\mathcal{N}\left(\mathbf{b}_{T}, \mathbf{r}_{T}-\mathbf{r}_{T}^{\prime}\right)-\mathcal{N}\left(\mathbf{b}_{T}, \mathbf{r}_{T}\right)-\right.} \\
\left.\mathcal{N}\left(\mathbf{b}_{T}, \mathbf{r}_{T}{ }^{\prime}\right) \mathcal{N}\left(\mathbf{b}_{T}, \mathbf{r}_{T}-\mathbf{r}_{T}{ }^{\prime}\right)\right]
\end{array}
$$

It is obvious that if the $b$-independent equation has a scaling solution that can be expressed in terms of a saturation scale $Q_{\mathrm{s}}$, then replacing it with an impact parameter dependent $Q_{\mathrm{s}}\left(\mathbf{b}_{T}\right)$ gives a solution of Eq. (18). This is effectively the approach used in Refs. [31, 54, 55]. The factorized ansatz Eq. (15) is not (unless the profile is a $\theta$ function) a solution of even this approximate equation. A factorized Gaussian profile for the proton dipole cross section, for example, does not approach the correct unitarity limit for $b \neq 0$. This is the main argument for including the impact parameter dependence in the saturation scale, not as a factorizable prefactor of the dipole cross section.

Because $b$ is the Fourier conjugate variable of $\Delta$, where $t=-\Delta^{2}$ is the momentum transfer squared between the incoming and outgoing proton, a Gaussian dependence of the impact parameter profile corresponds to an exponentially decreasing dependence of the dipole cross-section on $t$. This exponential behavior is widely observed for diffractive final states ${ }^{1}$. At smaller $Q^{2}$ (i.e. larger $r$ ) the impact parameter profile in the IPsat model is not exactly Gaussian, and diffractive peaks appear [20] at large $-t$. This is, however, in a region which is most likely unobservable because of the exponential suppression of the diffractive cross section. We emphasize (see also Ref. [19]) that specifying the full $\mathbf{b}_{T}$-dependence of the dipole cross section simultaneously determines both the normalization (" $\sigma_{0}$ ") of the cross section and the diffractive slope $B=\mathrm{d} \ln \sigma^{D} /\left.\mathrm{d} t\right|_{t=0}$.

In Fig. 3, we plot the inclusive and diffractive structure functions as a function of $b$, normalized to the same quantity at $b=0$. As has been noted previously, the inclusive distribution does not change significantly as $Q^{2}$ is varied. The $q \bar{q} g$ dipole contribution, at low $Q^{2}$, is significantly broader in $b$ than the inclusive distribution. This should be interpreted as its relative suppression at small $Q^{2}$ and small $b$, where the dipole cross section is close to the black disk limit and the $q \bar{q} g$ contribution vanishes, as is easily seen from Eq. (13).

Inclusive diffraction off nuclei is not very sensitive to the choice of impact parameter profiles in the proton. However, there are significant issues related to the impact parameter distribution of nuclei that are very relevant for diffraction, as we will see later. But before moving to scattering off nuclei we will first discuss the comparison of the IPsat and bCGC models to the HERA data on inclusive diffraction in ep scattering.

\section{COMPARISON TO HERA DATA}

An extensive comparison of the b-CGC and IPsat dipole models to the HERA data on exclusive vector meson production was performed in Ref. 22]. We compare our calculation to the HERA results on diffractive structure functions for $x_{\mathbb{P}}<0.01$, measured both using the rapidity gap method (ZEUS FPC [56] and H1 LRG [57]) and by measuring the recoil proton (ZEUS LPS [58] and H1 FPS [59]). Because the FPC and LRG data include events in which the proton has broken up, the crosssections measured for the process $e p \rightarrow e X Y$ are larger than the one measured for the process $e p \rightarrow e X p$. We scale down this data by a constant factor to correct for the proton dissociation contribution; the ZEUS FPC data by a factor of 1.45 and the H1 LRG data by 1.23. These factors are different due to the different cuts on $M_{Y}$, the mass of the proton system. Note that it is the FPSLPS data that correspond to our definition of diffractive events and to our formulae, as the proton should escape the collision intact. These correction factors were determined in the region $\beta \lesssim 0.7$ where data exists from both methods and then assumed to be independent of $\beta$; the

1 In contrast, the profile $T\left(\mathbf{b}_{T}\right)=2 \theta(R-b)$, leads to the $t$ distribution [28] $\sim 4\left|J_{1}(\sqrt{-t} R)\right|^{2} /\left(-t R^{2}\right) \approx 1+t R^{2} / 4+\mathcal{O}\left(t^{2}\right)$. 


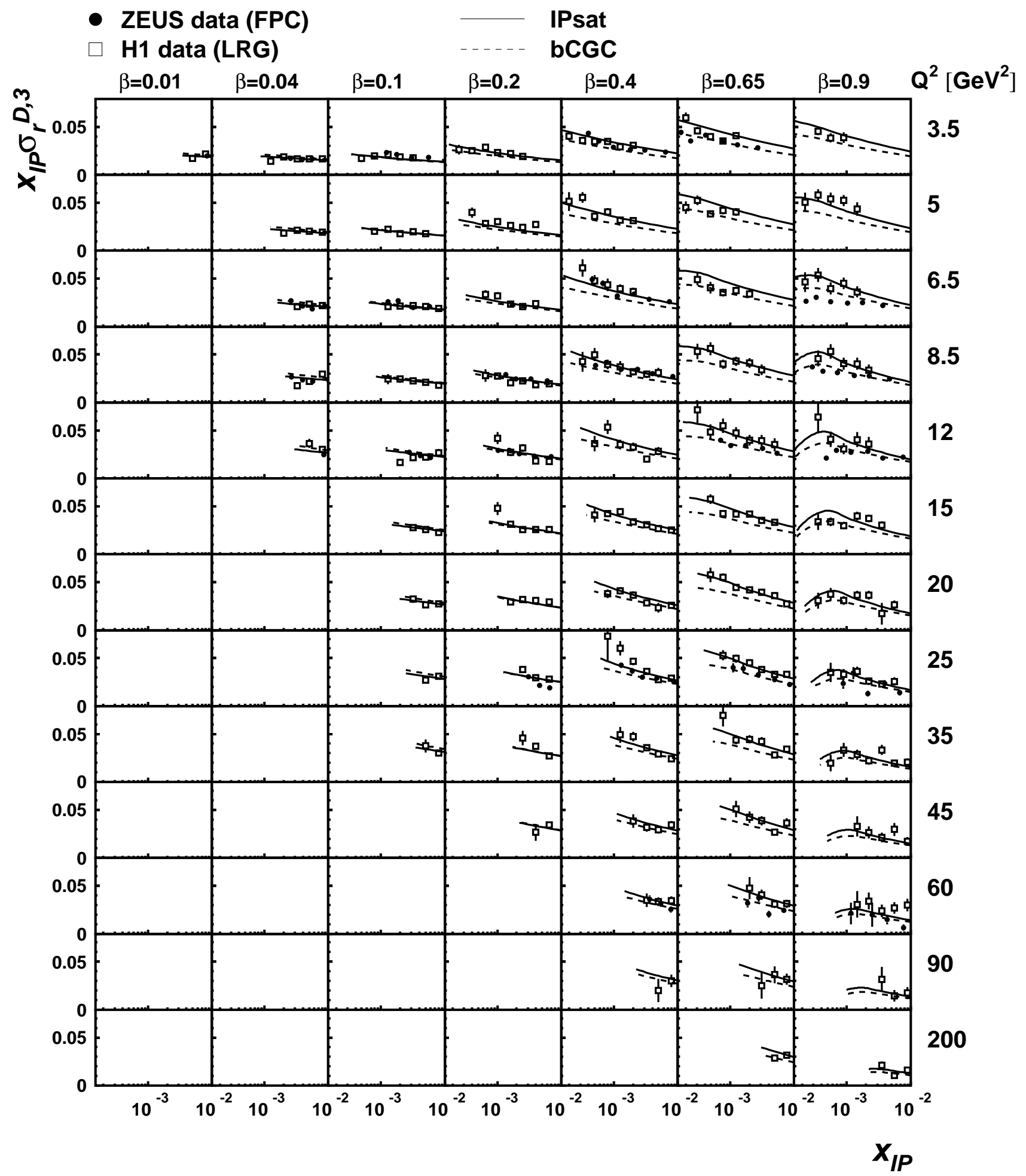

FIG. 4: Comparison of the IPsat and bCGC fits to HERA data on diffractive structure functions.

largest contributions to the $\chi^{2}$ of our analysis come from the large $\beta$ region where this assumption has not been tested experimentally. All the $\chi^{2}$-values quoted below are calculated per degree of freedom using statistical and systematical errors added in quadrature. The total num- ber of experimental points in the four data sets is 343 , of which 76 are from the FPS-LPS data.

The experimental data are presented in terms of the 
reduced cross section $\sigma_{r}^{D, 3}\left(\beta, x_{\mathbb{P}}, Q^{2}\right)$ :

$$
\begin{gathered}
\frac{d^{3} \sigma^{e p \rightarrow e X p}}{d x_{\mathbb{P}} d \beta d Q^{2}}=\frac{4 \pi \alpha_{e m}^{2}}{\beta Q^{4}}\left(1-y+\frac{y^{2}}{2}\right) \sigma_{r}^{D, 3}\left(\beta, x_{\mathbb{P}}, Q^{2}\right), \\
\sigma_{r}^{D, 3}=F_{T}^{D, 3}+\frac{2-2 y}{2-2 y+y^{2}} F_{L}^{D, 3} .
\end{gathered}
$$

with $y=Q^{2} /(s x)$ where $\sqrt{s}=318 \mathrm{GeV}$ is the total energy in the $e-p$ collision.

With the IPsat cross sections, for the combined dataset from ZEUS and $\mathrm{H} 1$ data both with and without identified protons we get $\chi^{2}=1.3$ for $\alpha_{\mathrm{s}}=0.14$ in Eqs. (10) and (12). For the bCGC cross section the fit is equally good and works with a larger value of the coupling: $\chi^{2}=1.3$ with $\alpha_{\mathrm{s}}=0.22$. These are the values of $\alpha_{\mathrm{s}}$ that we shall use for the respective models to evaluate nuclear diffractive structure functions in the next section. For the IPsat model the largest contribution to the $\chi^{2}$ comes from the rapidity gap method data at large $\beta$. The fit to only the LPS $\left(\chi^{2}=0.5\right.$ IPsat $)$ and FPS $\left(\chi^{2}=0.8\right)$ is much better. Considering just the LPS also accommodates a larger value of $\alpha_{\mathrm{s}}=0.21$ with still $\chi^{2}<1$. The fit for bCGC is more even among the datasets, but also there the $\mathrm{H} 1$ rapidity gap data has a larger $\chi^{2}=1.9$ than the other data sets. Our fit of these two models to a combination of the HERA datasets is presented in Fig. 4.

The fit to HERA data is better with a smaller $\alpha_{\mathrm{s}}$ than in Ref. [39]. Given the $b$-dependence described previously this is to be expected. The factorized $b$-dependence (Eq. (15)) used in earlier calculations of the diffractive structure function such as Refs. [8, 39] forces the $q \bar{q} g$ component to have the same impact parameter dependence as the $q \bar{q}$-component. In a more realistic description, as suggested by Fig. 3 the $q \bar{q} g$ component is sensitive to larger impact parameters and is thus larger; in order to fit the same data this must be compensated by multiplying it with a smaller factor of $\alpha_{\mathrm{s}}$.

\section{THE NUCLEAR DIFFRACTIVE STRUCTURE FUNCTION}

A straightforward generalization of the dipole formalism to nuclei is to introduce the coordinates of the individual nucleons $\left\{\mathbf{b}_{T i}\right\}$. In the IPsat model the replacement $T_{p}\left(\mathbf{b}_{T}\right) \rightarrow \sum_{i=1}^{A} T_{p}\left(\mathbf{b}_{T}-\mathbf{b}_{T i}\right)$ gives the dipole cross section

$$
\frac{\mathrm{d} \sigma_{\mathrm{dip}}^{A}}{\mathrm{~d}^{2} \mathbf{b}_{T}}=2\left[1-e^{-r^{2} F(x, r) \sum_{i=1}^{A} T_{p}\left(\mathbf{b}_{T}-\mathbf{b}_{T i}\right)}\right],
$$

where $F$ is defined in Eq. (3). The positions of the nucleons $\left\{\mathbf{b}_{T i}\right\}$ are distributed according to the Woods-Saxon distribution $T_{A}\left(\mathbf{b}_{T i}\right)$. We denote the average of an observable $\mathcal{O}$ over $\left\{\mathbf{b}_{T i}\right\}$ by

$$
\langle\mathcal{O}\rangle_{\mathrm{N}} \equiv \int \prod_{i=1}^{A} \mathrm{~d}^{2} \mathbf{b}_{T i} T_{A}\left(\mathbf{b}_{T i}\right) \mathcal{O}\left(\left\{\mathbf{b}_{T i}\right\}\right) .
$$

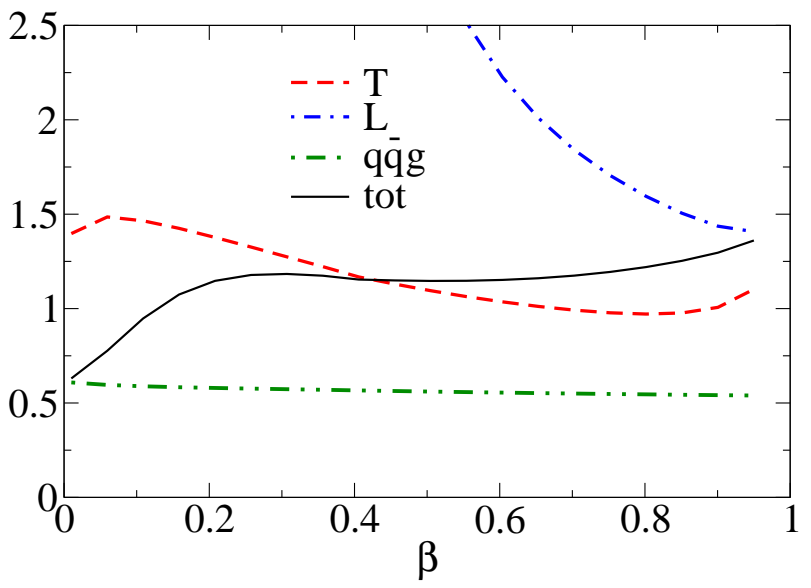

FIG. 5: The ratio $F_{2 A}^{\mathrm{D}}{ }^{x} /\left(A F_{2 p}^{\mathrm{D} x}\right)$ in the IPsat model for different components of the diffractive structure function $(x=$ $T, L, q \bar{q} g)$ as a function of $\beta$ for gold at $Q^{2}=5 \mathrm{GeV}^{2}$ and $x_{\mathbb{P}}=10^{-3}$ without nuclear breakup.

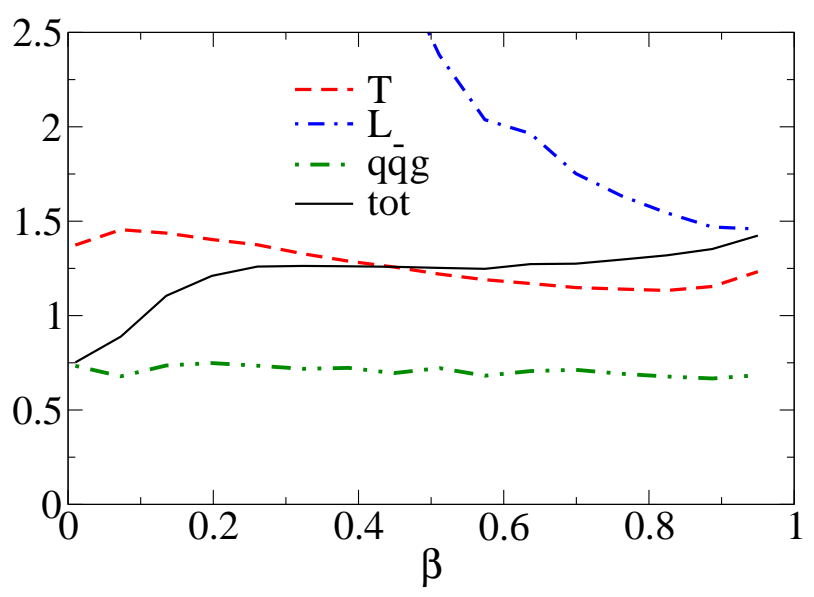

FIG. 6: Same as Fig. 5 but including nuclear breakup.

Here we have introduced the Woods-Saxon thickness function

$$
T_{A}\left(\mathbf{b}_{T}\right)=\int \mathrm{d} z \frac{C}{1+\exp \left[\left(\sqrt{\mathbf{b}_{T}^{2}+z^{2}}-R_{A}\right) / d\right]},
$$

which is normalized to unity $\int \mathrm{d}^{2} \mathbf{b}_{T} T_{A}\left(\mathbf{b}_{T}\right)=1$. The nuclear radius $R_{A}$ and surface diffuseness $d$ are measured from the electric charge distribution, their values can be found in Ref. [60]. The average differential dipole cross section is well approximated by [20]

$$
\left\langle\frac{\mathrm{d} \sigma_{\mathrm{dip}}^{A}}{\mathrm{~d}^{2} \mathbf{b}_{T}}\right\rangle_{\mathrm{N}} \approx 2\left[1-\left(1-\frac{T_{A}\left(\mathbf{b}_{T}\right)}{2} \sigma_{\mathrm{dip}}^{\mathrm{p}}\right)^{A}\right]
$$

where, for large $A$, the expression in parenthesis can be replaced by form [30]

$$
\exp \left(-\frac{A T_{A}\left(\mathbf{b}_{T}\right)}{2} \sigma_{\text {dip }}^{\mathrm{p}}\right) .
$$




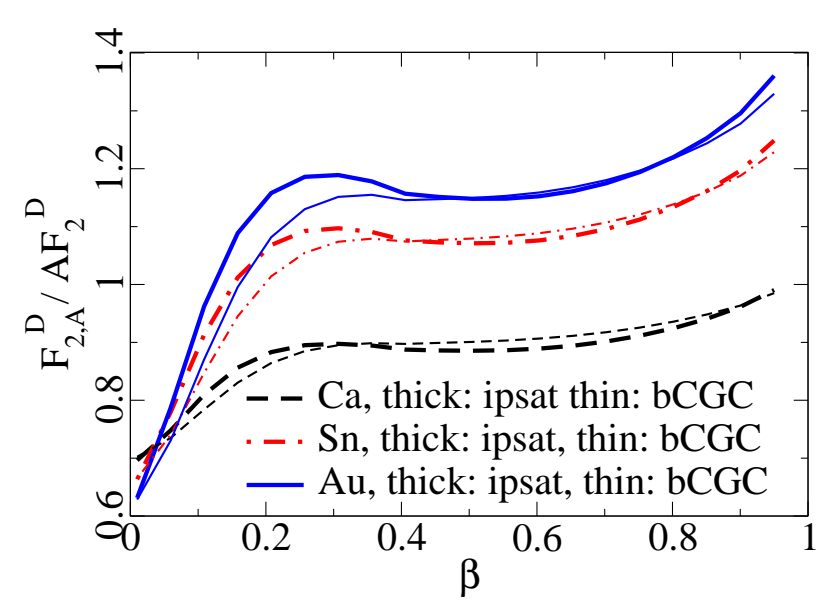

FIG. 7: The ratio $F_{2 A}^{\mathrm{D}} /\left(A F_{2 p}^{\mathrm{D}}\right)$ as a function of $\beta$ for $\mathrm{Ca}, \mathrm{Sn}$ and $\mathrm{Au}$ nuclei for $Q^{2}=5 \mathrm{GeV}^{2}$ and $x_{\mathbb{P}}=10^{-3}$. Results are for the "non breakup" case in the IPsat model (thick lines) and the bCGC model (thin lines).

All parameters of the model come from either fits of the model to $e p$-data or from the Woods-Saxon distribution; no additional parameters are introduced for $e A$ collisions.

The Glauber form (24) has a straightforward interpretation as the dipole scattering independently off the different nucleons. To see this explicitly denote $\frac{\mathrm{d} \sigma_{\mathrm{dip}}}{\mathrm{d}^{2} \mathbf{b}_{T}}\left(\mathbf{r}_{T}, \mathbf{b}_{T}\right)=2\left(1-S\left(\mathbf{r}_{T}, \mathbf{b}_{T}\right)\right)$, where the $S$-matrix element $S\left(\mathbf{r}_{T}, \mathbf{b}_{T}\right)$ is the amplitude for the dipole to not interact (elastically; the relation to the inclusive cross section is via the optical theorem) with the target. The $S$-matrix element for scattering off a nucleus is then given by $S_{A}\left(\mathbf{r}_{T}, \mathbf{b}_{T}\right)=\prod_{i=1}^{A} S_{p}\left(\mathbf{r}_{T}, \mathbf{b}_{T}-\mathbf{b}_{T i}\right)$ which, for the IPsat model, turns out to be equivalent to $T_{p}\left(\mathbf{b}_{T}\right) \rightarrow \sum_{i=1}^{A} T_{p}\left(\mathbf{b}_{T}-\mathbf{b}_{T i}\right)$. Note that in the form (24) there is no leading twist shadowing, i.e. in the large $Q^{2}$ or small $r$ limit $\sigma_{\text {dip }}^{A} \rightarrow A \sigma_{\text {dip }}^{\mathrm{p}}$, because in this limit $\sigma_{\text {dip }}^{\mathrm{p}} \sim r^{2}$ is small and one can expand the exponential.

The situation for the bCGC model is much more complicated, since the replacement $T_{p}\left(\mathbf{b}_{T}\right) \rightarrow \sum_{i=1}^{A} T_{p}\left(\mathbf{b}_{T}-\right.$ $\mathbf{b}_{T i}$ ) into the definition of the bCGC saturation scale (6) does not lead to the Glauber form (24). One could see this as a consequence of the "noncommutativity" of nuclear effects and high energy evolution: even if one assumes that for a particular $x$ and $\mathbf{r}_{T}$ a dipole interacts independently with the nucleons in a nucleus, this will not necessarily be the case for other rapidities and dipole sizes because the evolution sums up nonlinear interactions between the nucleons. Since it is not completely obvious how to introduce a nuclear dependence directly into the bCGC parametrization for the dipole cross section we will in this work use (24) for the bCGC model as well. A comparison of high energy evolution for protons and nuclei would be out of the scope of this work, see however Refs. 61, 62].

In Ref. 19], we showed that the nuclear dipole crosssections obtained in this manner gave a good (parameter
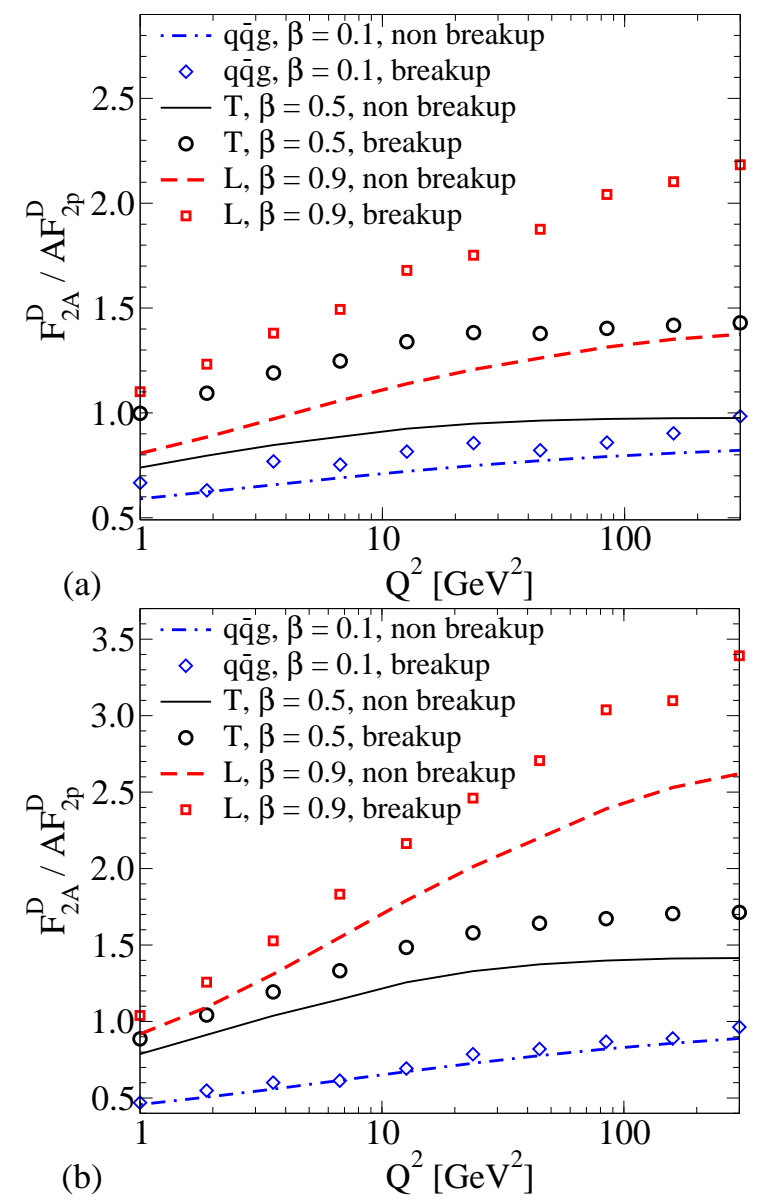

FIG. 8: The ratios $F_{2 A}^{\mathrm{D}}{ }^{x} /\left(A F_{2 p}^{\mathrm{D}^{x}}\right)$ at $x_{\mathbb{P}}=10^{-3}$ for different components of the diffractive structure function plotted as a function of $Q^{2}$. The components are evaluated where they are dominant: at $\beta=0.1$ for $q \bar{q} g, \beta=0.5$ for $T$ and $\beta=0.9$ for $L$. Results are in the IPsat model for both "breakup" and "no breakup" cases. (a) Ca nuclei, (b) Au nuclei.

free) agreement with the $x$ and $Q^{2}$ dependence of the NMC inclusive structure function data [63, 64] at small $x$. However, at the level of the accuracy of the data, it was not possible to distinguish between the IPsat and b-CGC models for the inclusive cross-section. We will now consider nuclear diffractive $(q \bar{q}$ and $q \bar{q} g)$ structure functions in the two dipole models. This is obtained by substituting the nuclear dipole cross-section (Eq. (24)) in Eqs. (7), (11) and (13).

It is very easy to break up a nucleus with a relatively small momentum transfer $|t| \gtrsim\left|t_{\text {min }}^{A}\right|$. However, for $\left|t_{\text {min }}^{A}\right| \lesssim|t| \lesssim\left|t_{\text {min }}^{p}\right|$, where $t_{\text {min }}^{p}$ is the minimum momentum transfer required to break up the proton, one can still have a nuclear diffractive event with a rapidity gap. For $|t| \gtrsim\left|t_{\min }^{A}\right|$, the "lumpiness" of the nucleus shows up as a proton-like tail $\left.\sim \exp \left\{C t R_{p}^{2}\right)\right\}$ of the $t$-distribution. In our formalism, if one requires that the nucleus stays completely intact, the average $\langle\cdot\rangle_{\mathrm{N}}$ in Eq. (22) must be performed at the amplitude level; the 
results are therefore proportional to $\left\langle\frac{\mathrm{d} \sigma_{\text {dip }}^{A}}{\mathrm{~d}^{2} \mathbf{b}_{T}}\right\rangle_{\mathrm{N}}^{2}$. One finds that in this case $\mathrm{d} \sigma^{D} / \mathrm{d} t$ falls off very rapidly as $\sim \exp \left\{C t R_{A}^{2}\right\}$. We refer to these (known as coherent diffraction) as "non breakup" events. Measuring the intact recoil nucleus at such a small $t$ experimentally at a future electron ion collider [65] is challenging. Also including events where the nucleus breaks up into color neutral constituents without filling the rapidity gap between the $q \bar{q}$ dipole and the nuclear fragmentation region (incoherent diffraction) corresponds to performing the average $\langle\cdot\rangle_{\mathrm{N}}$ over the cross section [66]; in this case, one performs the average $\left\langle\left(\frac{\mathrm{d} \sigma_{\text {dip }}^{A}}{\mathrm{~d}^{2} \mathbf{b}_{T}}\right)^{2}\right\rangle_{\mathrm{N}}$ instead. The difference between the two averaging procedures can be significant with increasing values of $t$ as shown explicitly in Ref. [19]. The "breakup" figures in our plots are a sum of these incoherent and coherent events.

In Figs. 5 and 6 we show the ratios of different components of the gold diffractive structure function to the proton one as a function of $\beta$. The $q \bar{q}$-components of the $F_{2 A}^{\mathrm{D}}$ are enhanced compared to $A$ times the proton diffractive structure functions. This is to be expected, because of the fact that in a gold nucleus the dipole cross section is, on average over the transverse area, closer to the unitarity limit than the proton - it is "blacker". The elastic scattering probability of a $q \bar{q}$ dipole is maximal in the "black" limit and the approach to it is quicker in a large nucleus. The $q \bar{q} g$ component, on the other hand, is suppressed for nuclei compared to the proton. This is due to the fact that in a nucleus the scattering amplitude is closer to the unitarity limit when the $q \bar{q} g$ component vanishes, as can be seen e.g. from Eq. (13). This leads to a nuclear suppression of the diffractive structure function in the small $\beta$ region, where the $q \bar{q} g$ component dominates. The net result of the different contributions is that $F_{2 A}^{\mathrm{D}}$, for a large range in $\beta$, is close to $A F_{2 p}^{\mathrm{D}}$.

In Fig. 7, we plot the total ratio as a function of $\beta$ for different nuclei in the "non breakup" case. As expected from our prior discussion, one sees a strong enhancement with $A$ for larger $\beta$ and likewise, a stronger suppression with $A$ at very small values of $\beta$. A comparison of the "breakup" versus "non breakup" cross-sections can be seen in Fig. 8 for the ratio of diffractive cross-sections as a function of $Q^{2}$. The results in Fig. 8 for the ratio of diffractive structure functions indicate that the diffractive cross-section in nuclei decrease more slowly for large $Q^{2}$ than in the proton.

In Figs. 9, 10 and 11 we show the $x_{\mathbb{P}}$ dependence of the nuclear modifications. For a fixed $Q^{2}=5 \mathrm{GeV}^{2}$ the nuclear enhancement of the $q \bar{q}$ components becomes smaller at smaller $x_{\mathbb{P}}$. This can easily be understood as an analogous effect to the $Q^{2}$-dependence plotted in Fig. 8, increasing $x_{\mathbb{P}}$ for a fixed $Q^{2}$ increases $Q^{2} / Q_{\mathrm{s}}{ }^{2}\left(x_{\mathbb{P}}\right)$ and has the same effect as increasing $Q^{2}$ at a fixed $x_{\mathbb{P}}$. In Fig. 10 we compare the $x_{\mathbb{P}}$-dependence in the IPsat and bCGC models. As was already observed in [19], the experimen-

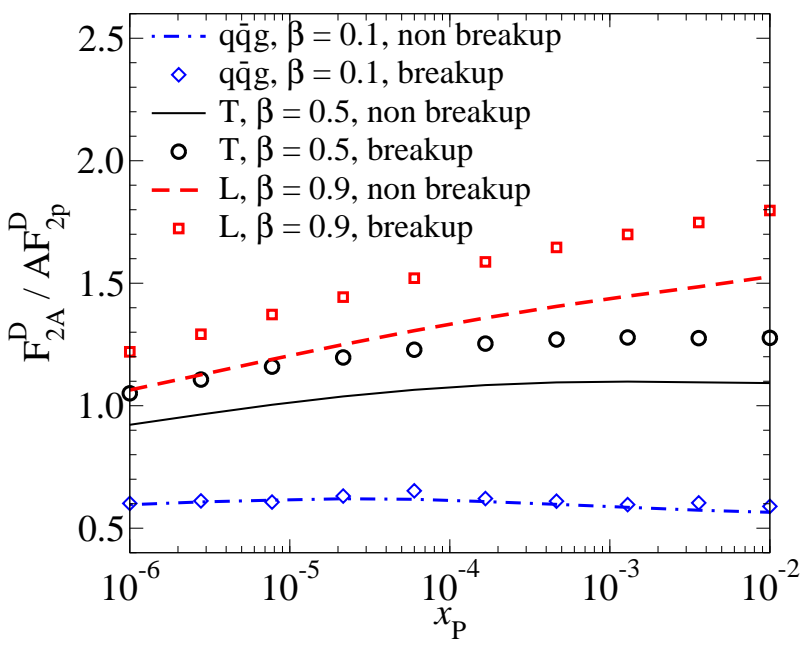

FIG. 9: The ratios $F_{2 A}^{\mathrm{D}}{ }^{x} /\left(A F_{2 p}^{\mathrm{D}^{x}}\right)$ for different components of the diffractive structure function plotted as a function of $x_{\mathbb{P}}$. The components are evaluated at $\beta=0.1$ for $q \bar{q} g, \beta=0.5$ for $T$ and $\beta=0.9$ for $L$. Results are in the IPsat model for Au nuclei both "breakup" and "no breakup" cases for $Q^{2}=5 \mathrm{GeV}^{2}$.

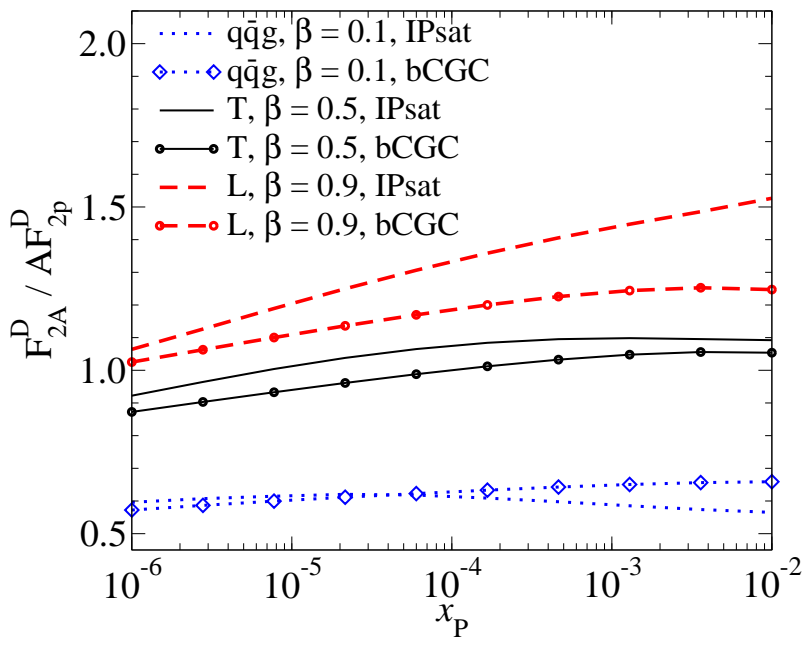

FIG. 10: The ratios $F_{2 A}^{\mathrm{D}}{ }^{x} /\left(A F_{2 p}^{\mathrm{D}}{ }^{x}\right)$ at $Q^{2}=5 \mathrm{GeV}^{2}$ for the IPsat and bCGC models as a function of $x_{\mathbb{P}}$, for the "no breakup" case. The components are evaluated at $\beta=0.1$ for $q \bar{q} g, \beta=0.5$ for $T$ and $\beta=0.9$ for $L$.

tal signature for the different evolution dynamics in the models in eA-scattering is mainly in the different $x_{\mathbb{P}}$ dependence in the nuclear modification factor. The result presented in Fig. 10 confirm this, although the effect is perhaps smaller than in the shadowing of the inclusive cross section. In Fig. 11 we compare, in the IPsat model, the nuclear modifications to $F_{T, q \bar{q}}^{D}$ at $\beta=0.5$ for $\mathrm{Ca}$, Sn and Au nuclei. One can see that the "non breakup" curves are much more sensitive to the nuclear species than the "breakup" ones.

In Fig. 12, the dependence of the longitudinal and transverse components of the diffractive structure func- 


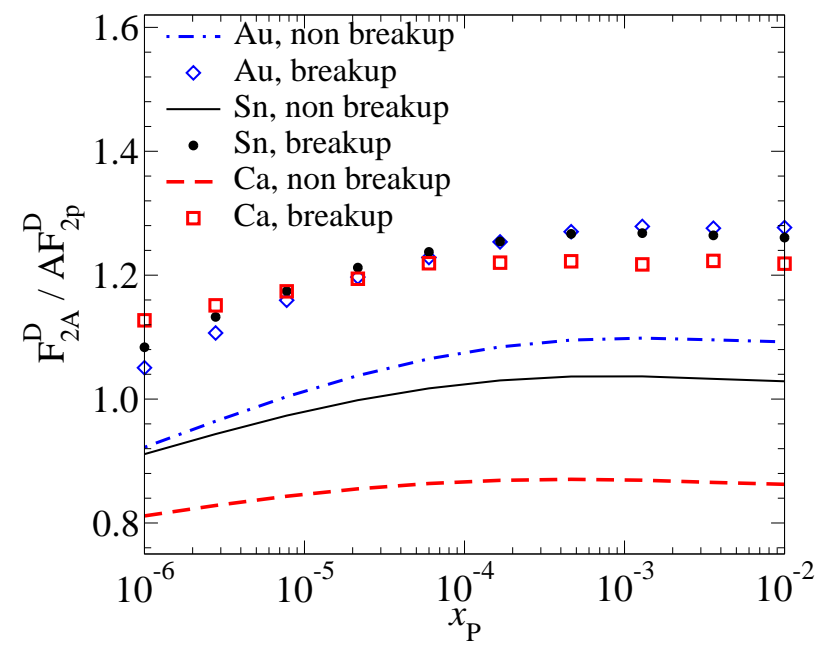

FIG. 11: $\quad F_{T, q \bar{q}, A}^{D} / A F_{T, q \bar{q}, p}^{D}$ at $Q^{2}=5 \mathrm{GeV}^{2}$ and $\beta=0.5$, as a function of $x_{\mathbb{P}}$ for $\mathrm{Au}$, Sn and Ca nuclei in the IPsat model. Both breakup and non-breakup cases are shown.

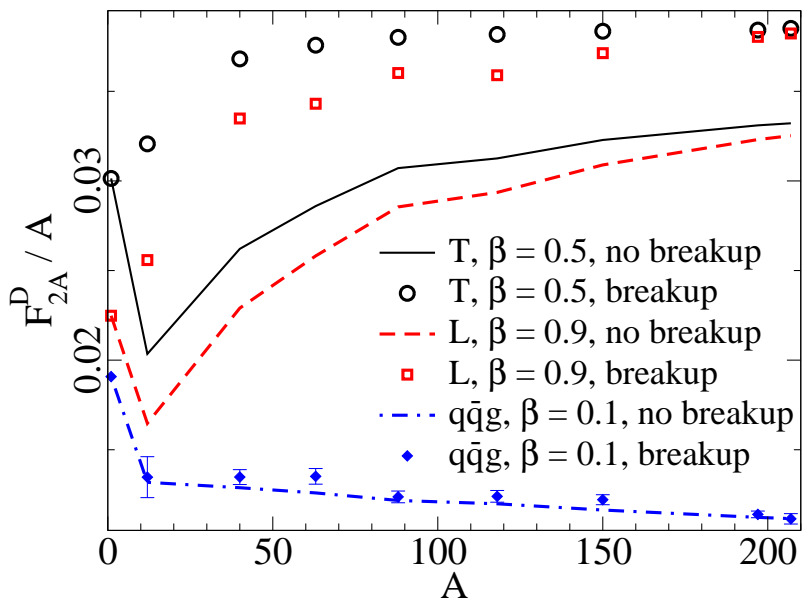

FIG. $12: \quad F_{T, q \bar{q}, A}^{D} / A$ at $\beta=0.5, F_{L, q \bar{q}, A}^{D} / A$ at $\beta=0.9$ and $F_{q \bar{q} g, A}^{D} / A$ at $\beta=0.1$ vs. A at $Q^{2}=5 G e V^{2}, x_{\mathbb{P}}=10^{-3}$. Both breakup and non-breakup cases are shown.

tion on nuclear size is shown for the "breakup" and "non breakup" cases. In the "breakup" case, one sees a very weak $A$ dependence In the coherent "non breakup" case, one first notes that the diffractive structure function first decreases up to atomic numbers $A \sim 10$, before beginning to rise. As noted in Ref. [19], this is due to the typical scattering amplitude for small nuclei actually being smaller than for a proton because of the diluteness of the nucleus. This leads to a suppression of coherent diffraction. The "breakup" case, on the other hand, can only be enhanced in nuclei. For gold nuclei, the cross sections in the "non breakup" case are about 15\% lower than in the "breakup" case.

Because of the different nuclear modifications in inclusive and diffractive scattering, the fraction of diffractive events in an experiment depends on the detailed kinematics and experimental coverage. Thus it is not straightforward to give a very precise general estimate for $\sigma_{\mathrm{D}} / \sigma_{\text {tot }}$ that would be observed in a generic high energy eA collider. A general order of magnitude argument would be as follows. For moderate values of $Q^{2}$ and large nuclei we expect a nuclear shadowing of the inclusive structure function by a factor $\sim 0.8[19]$. A typical nuclear enhancement of diffraction (at moderate values of $\beta \gtrsim 0.2$ ) is a factor of $\sim 1.2$ (see e.g. Fig. (7). Combining these we expect $\sigma_{\mathrm{D}} / \sigma_{\text {tot }}$ to be increased by a factor of $1.2 / 0.8=1.5$ compared to the proton. Thus from a typical ep fraction of $15 \%$ we expect $\sigma_{\mathrm{D}} / \sigma_{\text {tot }}$ to go up to $20 \%-25 \%$ at an eA collider.

\section{COMPARISONS AND CONCLUSIONS}

Finally we shall briefly compare our results to some other recent literature on diffraction in eA scattering. Several aspects of the treatment of nuclear diffraction here were discussed previously in the works of Nikolaev, Zakharov and Zoller [26] and of Frankfurt and Strikman [27] — these works may be consulted for earlier works in the literature as well. With regard to these early works, a key difference is the use of the explicit form of the $q \bar{q} g$ contributions to the diffractive structure functions given in Eq. (10) and (12). Further, we have focused on the predictions of Color Glass Condensate based models, that were previously constrained from fits to HERA data. In this paper, we have made explicit fits of these models to the HERA diffractive structure function data as well for the first time.

Turning to relatively more recent works in the literature, Gotsman, Levin, Lublinsky, Maor and Tuchin 30] use a parametrization of the impact parameter dependence that is similar to ours to compute the fraction of diffractive events in the total cross section. They see a modest enhancement compared to ep scattering in agreement with our results. As previously mentioned, they use a different method to calculate the $q \bar{q} g$ contribution but their results (see for example, Fig. 7 in Ref. [30]) seem to point towards its relative suppression in nuclei, in qualitative agreement with ours. On the other hand the energy dependence seems much stronger than our results indicate. Since the results of Ref. [30] are not presented in the form of diffractive structure functions (in particular the $\beta$-dependence is not calculated) a more detailed comparison is difficult. In Ref. 32] Levin and Lublinsky start from a Glauber-like parametrization very similar to our Eq. (25), but end up with a saturation scale depending on the nuclear size as $Q_{\mathrm{s}}{ }^{2} \sim A^{0.6}$ for moderately small values of $x \sim 10^{-3}$, which naturally leads to a much stronger $A$-dependence of $\sigma_{D} / \sigma_{\text {tot }}$ than our results.

In the work of Frankfurt, Guzey and Strikman 28, 29, 67], the nuclear diffractive structure functions are modelled starting from leading twist diffractive parton distributions in the proton. Although the terminology and theoretical framework are somewhat different, some comparisons can still be made. They find that $F_{2 A}^{\mathrm{D}} / A$ is 
suppressed compared to $F_{2 p}^{\mathrm{D}}$, as a function of $Q^{2}$, both at small $\beta=0.1$ (in agreement with our result) and in the transverse $q \bar{q}$ dominated region $\beta=0.5$ (which disagrees with our findings); this can be seen explicitly by comparing Fig. 5 in [67] and our Fig. 8 .

Kugeratski, Goncalves and Navarra [35, 68, 69] compute nuclear diffractive structure functions using the GBW framework [8], albeit with the IIM [21] dipole cross section. They extend the calculation to nuclei using a simple $A^{1 / 3}$-scaling of the saturation scale, the transverse area $\sigma_{0}$ and the diffractive slope $B_{D}$; the latter two are treated as independent parameters. They observe (see Figs. 4 and 6 in [35]) a similar pattern in the $\beta$-dependence as we do, namely that the nuclear $F_{2}^{\mathrm{D}}$ is relatively suppressed at small $\beta$. However, from their results one also deduces (note that the diffractive structure functions in [35] are normalized by $A^{4 / 3}$ which is not explicit in the text [70]) that $F_{2 A}^{\mathrm{D}} / A F_{2 p}^{\mathrm{D}}$ is of the order of $1 / 4$, which is much smaller than our result $\sim 1$ (see Fig. 7). We conjecture that these differences arise because, unlike in our discussion, $\sigma_{0}$ and $B_{D}$ are treated as independent parameters in Ref. [35], with values that are not necessarily consistent with each other.

Measuring diffractive events in a high energy eA collider would yield significant insight into the physics of high parton densities. The experimental measurements of inclusive diffraction are encoded in diffractive struc- ture functions that depend on the kinematical variables of the process. We have argued that it is possible to make a large number of detailed theory predictions for nuclear diffractive structure functions. Comparing these to experimental measurements would provide a powerful handle to understanding the behavior of QCD at high energy. In this paper we have presented some of these theory predictions, within two particular models for the high energy wavefunction. One (IPsat) is constructed to combine the correct DGLAP limit at large $Q^{2}$ with unitarization at small $Q^{2}$. The other (bCGC) is a parametrization of the leading order BK dynamics. We have also emphasized the importance of including in the calculations a realistic description of the impact parameter dependence in the nucleus, without which an actual comparison to experimental data is not very useful.

\section{Acknowledgments}

We thank M. Strikman, M. S. Kugeratski, V. P. Goncalves and F. S. Navarra for discussions. RV's research is supported by DOE Contract No. DE-AC0298CH10886 and CM's research is supported by the European Commission under the FP6 program, contract No. MOIF-CT-2006-039860.
[1] ZEUS Collaboration, M. Derrick et. al., Phys. Lett. B315 (1993) 481.

[2] H1 Collaboration, T. Ahmed et. al., Nucl. Phys. B429 (1994) 477.

[3] H1 Collaboration, C. Adloff et. al., Z. Phys. C76 (1997) 613 arXiv:hep-ex/9708016.

[4] ZEUS Collaboration, J. Breitweg et. al., Eur. Phys. J. C1 (1998) 81 arXiv:hep-ex/9709021.

[5] A. M. Stasto, K. Golec-Biernat and J. Kwiecinski, Phys. Rev. Lett. 86 (2001) 596 arXiv:hep-ph/0007192].

[6] C. Marquet and L. Schoeffel, Phys. Lett. B639 (2006) 471 arXiv:hep-ph/0606079.

[7] F. Gelis, R. Peschanski, G. Soyez and L. Schoeffel, Phys. Lett. B647 (2007) 376 arXiv:hep-ph/0610435.

[8] K. Golec-Biernat and M. Wusthoff, Phys. Rev. D60 (1999) 114023 arXiv:hep-ph/9903358.

[9] K. Golec-Biernat and M. Wusthoff, Phys. Rev. D59 (1999) 014017 arXiv:hep-ph/9807513.

[10] L. V. Gribov, E. M. Levin and M. G. Ryskin, Phys. Rept. 100 (1983) 1.

[11] J. Bartels, K. Golec-Biernat and H. Kowalski, Phys. Rev. D66 (2002) 014001 arXiv:hep-ph/0203258.

[12] L. D. McLerran and R. Venugopalan, Phys. Rev. D49 (1994) 2233 arXiv:hep-ph/9309289.

[13] L. D. McLerran and R. Venugopalan, Phys. Rev. D49 (1994) 3352 arXiv:hep-ph/9311205.

[14] L. D. McLerran and R. Venugopalan, Phys. Rev. D50 (1994) 2225 arXiv:hep-ph/9402335.

[15] E. Iancu and R. Venugopalan, arXiv:hep-ph/0303204

[16] H. Weigert, Prog. Part. Nucl. Phys. 55 (2005) 461
arXiv:hep-ph/0501087.

[17] A. H. Mueller, Nucl. Phys. B335 (1990) 115.

[18] N. N. Nikolaev and B. G. Zakharov, Z. Phys. C49 (1991) 607.

[19] H. Kowalski, T. Lappi and R. Venugopalan, Phys. Rev. Lett. 100 (2008) 022303 arXiv:0705.3047 [hep-ph].

[20] H. Kowalski and D. Teaney, Phys. Rev. D68 (2003) 114005 arXiv:hep-ph/0304189.

[21] E. Iancu, K. Itakura and S. Munier, Phys. Lett. B590 (2004) 199 arXiv:hep-ph/0310338.

[22] H. Kowalski, L. Motyka and G. Watt, Phys. Rev. D74 (2006) 074016 arXiv:hep-ph/0606272.

[23] E. Iancu, K. Itakura and L. McLerran, Nucl. Phys. A708 (2002) 327 arXiv:hep-ph/0203137.

[24] J. R. Forshaw, R. Sandapen and G. Shaw, JHEP 11 (2006) 025 arXiv:hep-ph/0608161.

[25] N. Nikolaev and B. G. Zakharov, Z. Phys. C53 (1992) 331.

[26] N. N. Nikolaev, B. G. Zakharov and V. R. Zoller, Z. Phys. A351 (1995) 435.

[27] L. L. Frankfurt and M. I. Strikman, Phys. Lett. B382 (1996) 6.

[28] L. Frankfurt, V. Guzey, M. McDermott and M. Strikman, arXiv:hep-ph/0104252.

[29] L. Frankfurt, V. Guzey, M. McDermott and M. Strikman, JHEP 02 (2002) 027 arXiv:hep-ph/0201230.

[30] E. Gotsman, E. Levin, M. Lublinsky, U. Maor and K. Tuchin, Phys. Lett. B492 (2000) 47 arXiv:hep-ph/9911270. 
[31] E. Levin and M. Lublinsky, Nucl. Phys. A696 (2001) 833 arXiv:hep-ph/0104108.

[32] E. Levin and M. Lublinsky, Nucl. Phys. A712 (2002) 95 arXiv:hep-ph/0207374.

[33] B. Z. Kopeliovich, A. Schafer and A. V. Tarasov, Phys. Rev. D62 (2000) 054022 arXiv:hep-ph/9908245.

[34] B. Z. Kopeliovich, B. Povh and I. Schmidt, Nucl. Phys. A782 (2007) 24 arXiv:hep-ph/0607337.

[35] M. S. Kugeratski, V. P. Goncalves and F. S. Navarra, Eur. Phys. J. C46 (2006) 413 arXiv:hep-ph/0511224.

[36] Y. V. Kovchegov and E. Levin, Nucl. Phys. B577 (2000) 221 arXiv:hep-ph/9911523.

[37] Y. Hatta, E. Iancu, C. Marquet, G. Soyez and D. N. Triantafyllopoulos, Nucl. Phys. A773 (2006) 95 arXiv:hep-ph/0601150.

[38] M. Wusthoff, Phys. Rev. D56 (1997) 4311 arXiv:hep-ph/9702201.

[39] C. Marquet, Phys. Rev. D76 (2007) 094017 arXiv:0706.2682 [hep-ph].

[40] I. Balitsky, Nucl. Phys. B463 (1996) 99 arXiv:hep-ph/9509348.

[41] Y. V. Kovchegov, Phys. Rev. D60 (1999) 034008 arXiv:hep-ph/9901281.

[42] Y. V. Kovchegov, Phys. Rev. D61 (2000) 074018 arXiv:hep-ph/9905214.

[43] S. Munier and A. Shoshi, Phys. Rev. D69 (2004) 074022 arXiv:hep-ph/0312022.

[44] J. Bartels, H. Jung and M. Wusthoff, Eur. Phys. J. C11 (1999) 111 arXiv:hep-ph/9903265.

[45] Y. V. Kovchegov, Phys. Rev. D64 (2001) 114016 arXiv:hep-ph/0107256.

[46] C. Marquet, Nucl. Phys. B705 (2005) 319 arXiv: hep-ph/0409023.

[47] K. J. Golec-Biernat and C. Marquet, Phys. Rev. D71 (2005) 114005 arXiv:hep-ph/0504214.

[48] J. Bartels, J. R. Ellis, H. Kowalski and M. Wusthoff, Eur. Phys. J. C7 (1999) 443 arXiv:hep-ph/9803497.

[49] E. Levin and M. Lublinsky, Eur. Phys. J. C22 (2002) 647 arXiv:hep-ph/0108239.

[50] L. Frankfurt, V. Guzey, M. McDermott and M. Strikman, Phys. Rev. Lett. 87 (2001) 192301 arXiv:hep-ph/0104154.

[51] L. Frankfurt, M. Strikman and C. Weiss, Ann. Rev.
Nucl. Part. Sci. 55 (2005) 403 arXiv:hep-ph/0507286.

[52] Y. Hatta and A. H. Mueller, Nucl. Phys. A789 (2007) 285 arXiv:hep-ph/0702023.

[53] K. J. Golec-Biernat and A. M. Stasto, Nucl. Phys. B668 (2003) 345 arXiv:hep-ph/0306279.

[54] M. Lublinsky, E. Gotsman, E. Levin and U. Maor, Nucl. Phys. A696 (2001) 851 arXiv:hep-ph/0102321.

[55] E. Gotsman, E. Levin, M. Lublinsky and U. Maor, Eur. Phys. J. C27 (2003) 411 arXiv:hep-ph/0209074.

[56] ZEUS Collaboration, S. Chekanov et. al., Nucl. Phys. B713 (2005) 3 arXiv:hep-ex/0501060.

[57] H1 Collaboration, A. Aktas et. al., Eur. Phys. J. C48 (2006) 715 arXiv:hep-ex/0606004.

[58] ZEUS Collaboration, S. Chekanov et. al., Eur. Phys. J. C38 (2004) 43 arXiv:hep-ex/0408009.

[59] H1 Collaboration, A. Aktas et. al., Eur. Phys. J. C48 (2006) 749 arXiv:hep-ex/0606003.

[60] C. W. De Jager, H. De Vries and C. De Vries, Atom. Data Nucl. Data Tabl. 36 (1987) 495.

[61] A. H. Mueller, Nucl. Phys. A724 (2003) 223 arXiv:hep-ph/0301109.

[62] J. L. Albacete, N. Armesto, J. G. Milhano, C. A. Salgado and U. A. Wiedemann, Phys. Rev. D71 (2005) 014003 arXiv:hep-ph/0408216.

[63] NMC Collaboration, P. Amaudruz et. al., Nucl. Phys. B441 (1995) 3 arXiv:hep-ph/9503291.

[64] NMC Collaboration, M. Arneodo et. al., Nucl. Phys. B481 (1996) 3.

[65] A. Deshpande, R. Milner, R. Venugopalan and W. Vogelsang, Ann. Rev. Nucl. Part. Sci. 55 (2005) 165 arXiv:hep-ph/0506148.

[66] Y. V. Kovchegov and L. D. McLerran, Phys. Rev. D60 (1999) 054025 arXiv:hep-ph/9903246.

[67] L. Frankfurt, V. Guzey and M. Strikman, Phys. Lett. B586 (2004) 41 arXiv:hep-ph/0308189.

[68] V. P. Goncalves, M. S. Kugeratski, M. V. T. Machado and F. S. Navarra, Phys. Lett. B643 (2006) 273 arXiv:hep-ph/0608063.

[69] E. R. Cazaroto, F. Carvalho, V. P. Goncalves and F. S. Navarra, arXiv:0805.1255 [hep-ph].

[70] V. P. Goncalves, M. S. Kugeratski and F. S. Navarra. Private communication. 